\title{
QSO host galaxies and environments in the ELT era
}

\author{
Simona Paiano ${ }^{\mathrm{a}}$, Renato Falomo ${ }^{\mathrm{a}}$, Michela Uslenghi $^{\mathrm{b}}$, Carmelo Arcidiacono $^{\mathrm{c}}$, Daniela \\ Fantinel $^{\mathrm{a}}$, and Roberto Ragazzoni ${ }^{\mathrm{a}}$ \\ ${ }^{a}$ INAF - Osservatorio Astronomico di Padova, Italy \\ ${ }^{b}$ INAF - Istituto di Astrofisica Spaziale e Fisica Cosmica di Milano, Italy \\ ${ }^{\mathrm{c}} \mathrm{INAF}$ - Osservatorio Astronomico di Bologna, Italy
}

\begin{abstract}
Quasars represent our main lighthouses to explore the high redshift Universe and to investigate the processes of formation of galaxies and their central supermassive black holes. The characterization of the key parameters of quasar hosts as the galaxy mass, size, morphology and their close environments are hindered by the limited resolution and sensitivity of the present instrumentation. We investigate here the expected capabilities of future observations obtained with near-IR Adaptive Optics imaging cameras foreseen for Extremely Large Telescopes. Detailed simulations are presented to evaluate the accuracy of those observations under different observing conditions.
\end{abstract}

Keywords: QSO host galaxy, Adaptive optics, Extremely Large Telescopes.

\section{INTRODUCTION}

Quasars (QSOs) represent our main lighthouses to explore the high redshift Universe and to investigate the processes of formation of galaxies and their central supermassive black holes. The discovery of a relationship between the central black hole and the properties of their host galaxies is a fundamental ingredient for a comprehensive interpretation of the structure and evolution of the galaxies. Of particular relevance is the understanding of how this relationship evolves over the cosmic time and it can offer important clues for the study of the formation processes of galaxies and their central massive black holes. Another key issue is to explore the immediate environment of the galaxies that host powerful active nuclei to search for signature of interaction and/or merging that is considered a fundamental ingredient for triggering and fueling the nuclear activity (e.g. Refs. 1, 2, 3). The characterization of the key parameters as the QSO host galaxy mass, size, morphology and their close environments are hindered by the limited resolution and sensitivity of the present instrumentation (in the case of Hubble Space Telescope (HST) the best resolution is $\sim 15$ mas).

The measurement of the properties of the QSO host galaxies is increasingly more challenging at high redshift. This is because the contrast between the bright central nucleus and the starlight from the host galaxy critically depends on the size and shape of the latter compared with that of the point spread function (PSF). Observations from space with the HST can indeed provide an excellent narrow PSF, but because of the faintness of high redshift host galaxies, these results are usually limited by the small collecting area of the telescope and the limited performances in the near-IR. The future James Webb Space Telescope (JWST, https://www.jwst.nasa.gov/) expected to be operative by end of this decade would yield a further significant improvement to these studies because of its large aperture and optimization for NIR frequencies. In spite of that, the resolution of the images obtainable with NIRCam for JWST (pixel size $\sim 30$ mas) is still smaller with respect to that foreseen of future ground based Extreme Large Telescopes (ELT) (pixel size of 2 - 4 mas), assisted by adaptive optics (AO) module. At high redshift $(\mathrm{z}>1)$ the size of the galaxies hosting quasars are smaller than 1 arcsec and their faint starlight is hidden by that of the bright nucleus. It is therefore envisaged that only in the ELT era it will be possible to detect and characterize very small and faint features in the host galaxies of distant quasars.

Further author information: (Send correspondence to Simona Paiano)

Simona Paiano: E-mail: simona.paiano@oapd.inaf.it

Renato Falomo: E-mail: renato.falomo@oapd.inaf.it 
We investigate here the foreseen capabilities of future observations obtained with near-IR AO imaging cameras for ELTs. In particular, the case of Multi-Conjugate Adaptive Optics (MCAO) observations of distant quasars obtainable with the post-focal AO module MAORY (Multi-conjugate Adaptive Optics RelaY) and the near-IR camera MICADO provided for the European Extremely Large Telescope (EELT, http://www.eso.org/sci/facilities/eelt/), is investigated and compared with expected images from JWST and current observations AO images at 8-m telescopes.

\section{SIMULATION OF QSO IMAGES}

We use a preliminary evaluation of the PSF for the MCAO module of MAORY [4] to probe the capabilities of EELT images to detect morphology of host galaxy of QSOs and signature of interactions. Since in June 2017 the final MAORY Phase B MCAO PSFs [5] were not released, but the Phase B SCAO PSFs were available within the MAORY consortium, we decide to merge the 2012 release of the PSF in the phase-A study with the SCAO Phase B PSF. This resulted in the addition of the pupil segmentation effects (M1 primary shape) to the Phase-A PSF. Actually, the comparison of the two images show that pupil segmentation effects were not considered on the 2012 MCAO release*. This effect looks important in terms of contrast both in the inner and outer region of the PSF. We subtracted the Airy diffraction pattern (see Fig. 1) corresponding to a circular pupil of $42 \mathrm{~m}$ with a central obstruction with 0.27 ratio, in the whole Ks filter at 2.12 micron. We then added the diffraction pattern expected from the E-ELT 39.5m pupil on the PSF residual, taking care to balance the energy removed on the first step. The original 512px $\times 512 \mathrm{px}$ MCAO PSF showed a pattern of a concentric squares corresponding to the power of 2 dimension 128 and 256, see Fig. 1. These two patterns are residuals of numerical approximation and are not true optical features. We extrapolated the power law corresponding to the seeing halo in the original central 128x128 region of the MCAO PSF overwriting the external part of the PSF.

DIFFRACTION

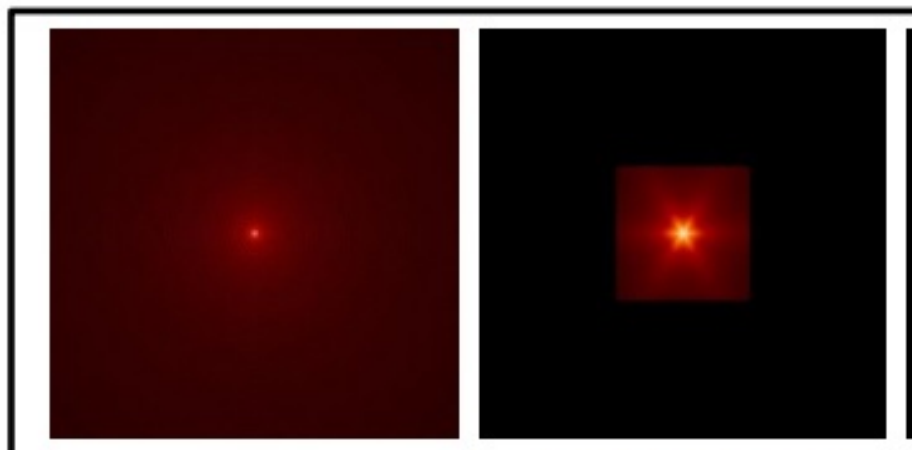

Airy
FITTING + ALIASING ERRORS

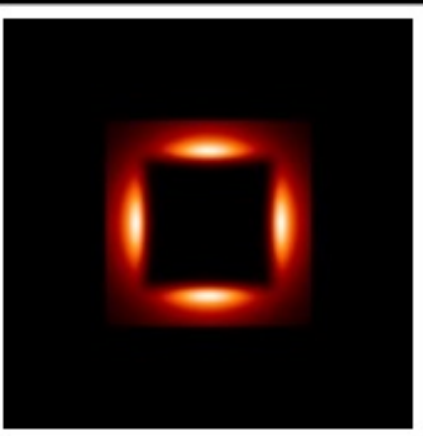

Moffat $\times$ mask
SEEING

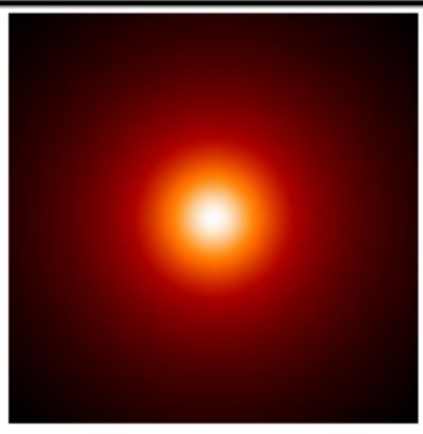

Moffat

Figure 1. The components of the MAORY Phase A PSF taken from [6].

The resulted PSF is shown in Fig. 2 (right panel) and it is compared with the MAORY Phase-A PSF (left panel). A more quantitative comparison is show in Fig. 3, where we plot the average radial brightness profile (left panel) and the encircled energy as function of the radius (right panel) for the two different PSFs.

The QSO image simulations are performed using the Advanced Exposure Time Calculator tool (AETC, http://aetc.oapd.inaf.it/ ). Throughput and sky and environment background as defined by EELT + MICADO + MAORY configuration: these include sky and environment background, statistical and read out noise, and appropriate convolution of the galaxy models with the PSF. Both statistical and fixed pattern noise are also included. To simulate the host galaxies of the QSO we consider images of low redshift galaxies secured by HST ACS as template and add a point source for the bright nucleus, fitted by a pure stellar PSF function. The galaxy

\footnotetext{
${ }^{*}$ See details in http://www.eso.org/sci/meetings/2015/EriceSchool2015/Schreibermapping_PSF.pdf
} 

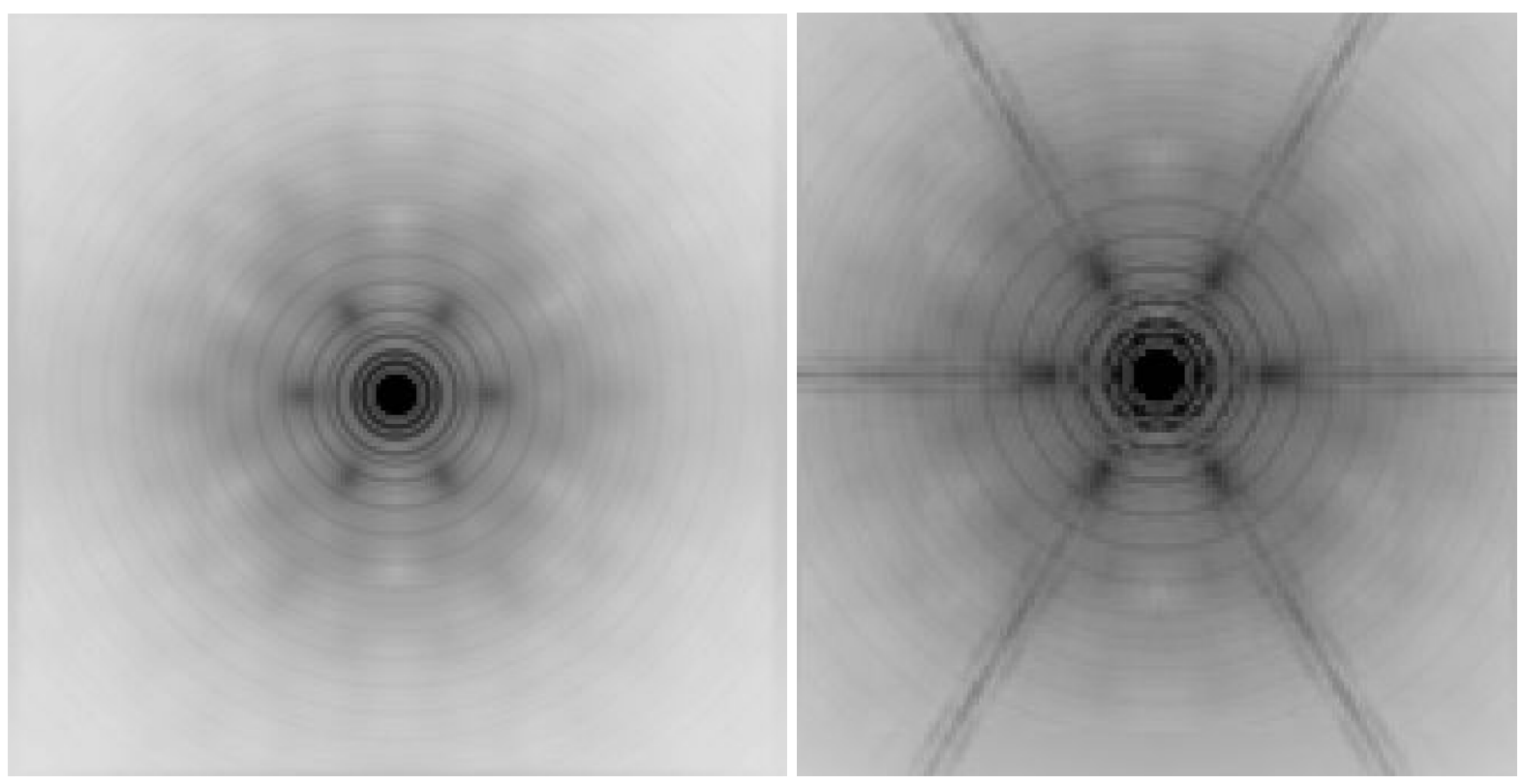

Figure 2. Image of the MAORY Phase A PSF released in 2012 (left panel), and the upgraded PSF used in this work (right panel, see details in the text).
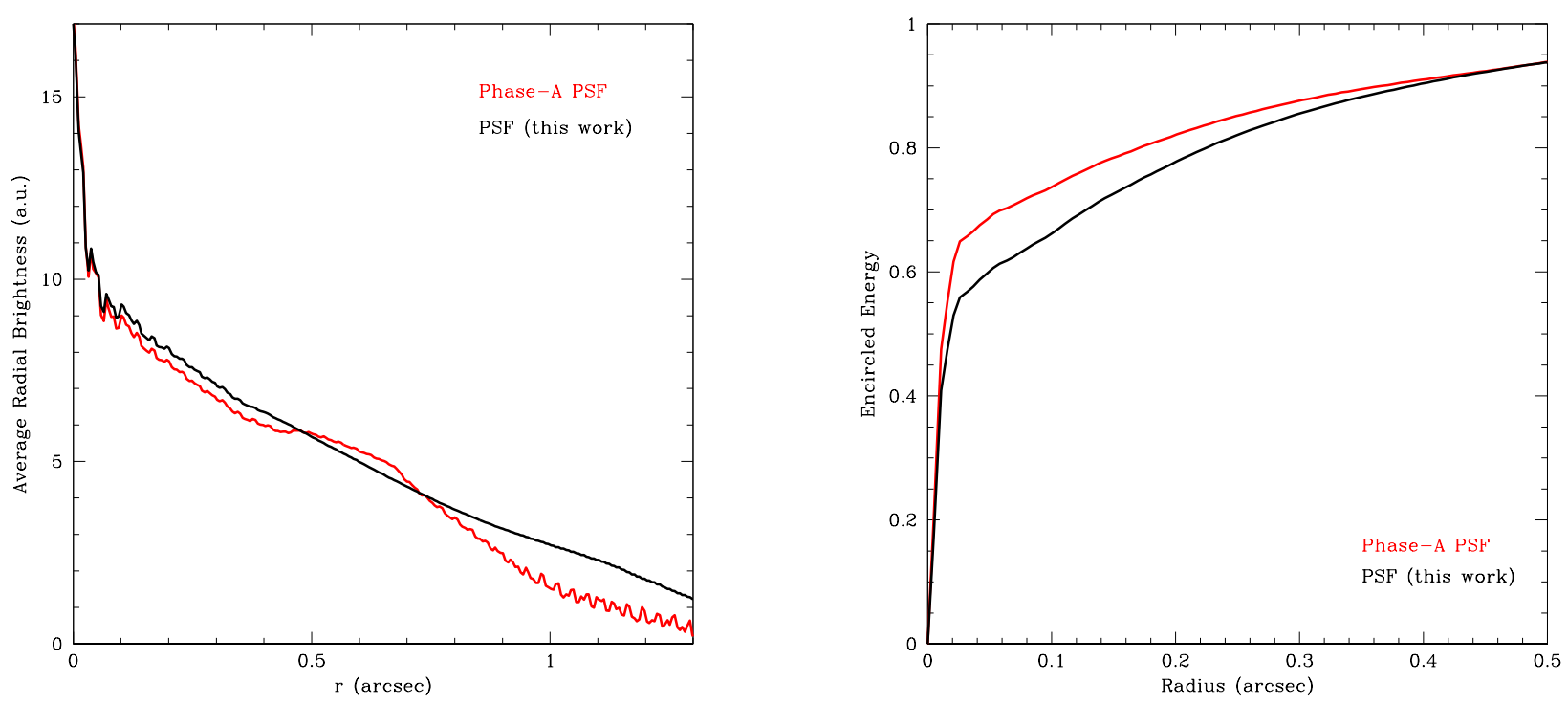

Figure 3. Left Panel: Average radial brightness profile of the MAORY Phase-A PSF (red curve) and the PSF used in this work (black curve). Right panel: Encircled Energy as function of the radius for the two different PSFs.

template is convolved with the proper PSF and then scaled to the flux and the angular size that is expected for a QSO at a given redshift.

We are carrying out several simulations of high redshift QSO with different nucleus to host brightness ratio combinations, in order to evaluate the capabilities of MICADO@EELT, to characterize the structural and photometric properties of their host galaxies and of the immediate environments. We varied the apparent magnitude 
of the nucleus from 15 to 20 in the Ks-band, and for host galaxy we used values from the magnitude of the nucleus to 23. In Fig. 4 and Fig. 5, we show two representative examples of a QSO at $\mathrm{z}=1$ and $\mathrm{z}=3$, where the source parameters and the galaxy templates used are reported in Table 1. For comparison we also performed similar simulations as would be obtained by NIRCam on board of JWST using the specifications given in http://jwstetc.stsci.edu/etc/) and by a MCAO system (alike MAD@VLT, see 7) on an 8-m ground telescope for the same targets and same exposure times.

Table 1. Parameters of the sources used in the simulations reported in Fig. 4 and Fig. 5

\begin{tabular}{|c|c|c|c|}
\hline QSO $1(z=1)$ & Ks mag & Size (") & Galaxy Template \\
\hline Nucleus & 18 & $*$ & $*$ \\
\hline Host galaxy & 19 & 0.3 & NGC 6050 \\
\hline \multicolumn{4}{|l|}{ QSO $2(\mathrm{z}=3)$} \\
\hline Nucleus & 21 & $*$ & $*$ \\
\hline Host galaxy & 21 & 0.1 & NGC 1309 \\
\hline $1^{\text {st }}$ Companion galaxy & 21.5 & 0.1 & M 82 at $0.15 "$ from the nucleus \\
\hline $2^{\text {nd }}$ Companion galaxy & 21.5 & 0.1 & M 82 at $0.35 "$ from the nucleus \\
\hline
\end{tabular}

\section{REFERENCES}

[1] Di Matteo, T., Springel, V., and Hernquist, L., "Energy input from quasars regulates the growth and activity of black holes and their host galaxies," Nature 433, 604-607 (Feb. 2005).

[2] Hopkins, P. F., Hernquist, L., Cox, T. J., and Kereš, D., "A Cosmological Framework for the Co-Evolution of Quasars, Supermassive Black Holes, and Elliptical Galaxies. I. Galaxy Mergers and Quasar Activity," ApJS 175, 356-389 (Apr. 2008).

[3] Heckman, T. M. and Best, P. N., "The Coevolution of Galaxies and Supermassive Black Holes: Insights from Surveys of the Contemporary Universe," ARA\&A 52, 589-660 (Aug. 2014).

[4] Arcidiacono, C., Schreiber, L., Bregoli, G., Diolaiti, E., Foppiani, I., Cosentino, G., Lombini, M., Butler, R. C., and Ciliegi, P., "End to end numerical simulations of the MAORY multiconjugate adaptive optics system," in [Adaptive Optics Systems IV], Proc. SPIE 9148, 91486F (Aug. 2014).

[5] Diolaiti, E., Arcidiacono, C., Bregoli, G., Butler, R. C., Lombini, M., Schreiber, L., Baruffolo, A., Basden, A., Bellazzini, M., Cascone, E., Ciliegi, P., Cortecchia, F., Cosentino, G., De Caprio, V., De Rosa, A., Dipper, N., Esposito, S., Foppiani, I., Giro, E., Morgante, G., Myers, R., Patru, F., Ragazzoni, R., Riccardi, A., Riva, M., Zerbi, F. M., Casali, M., Delabre, B., Hubin, N., Kerber, F., Le Louarn, M., Marchetti, E., Ramsay, S., Stroebele, S., and Vernet, E., "Preparing for the phase B of the E-ELT MCAO module project," in [Adaptive Optics Systems IV], Proc. SPIE 9148, 91480Y (Aug. 2014).

[6] Schreiber, L., La Camera, A., Prato, M., and Diolaiti, E., "Point Spread Function extraction in crowded fields using blind deconvolution," in [Proceedings of the Third AO4ELT Conference], Esposito, S. and Fini, L., eds., 78 (Dec. 2013).

[7] Liuzzo, E., Falomo, R., Paiano, S., Treves, A., Uslenghi, M., Arcidiacono, C., Baruffolo, A., Diolaiti, E., Farinato, J., Lombini, M., Moretti, A., Ragazzoni, R., Brast, R., Donaldson, R., Kolb, J., Marchetti, E., and Tordo, S., "MAD Adaptive Optics Imaging of High-luminosity Quasars: A Pilot Project," AJ 152, 38 (Aug. 2016). 

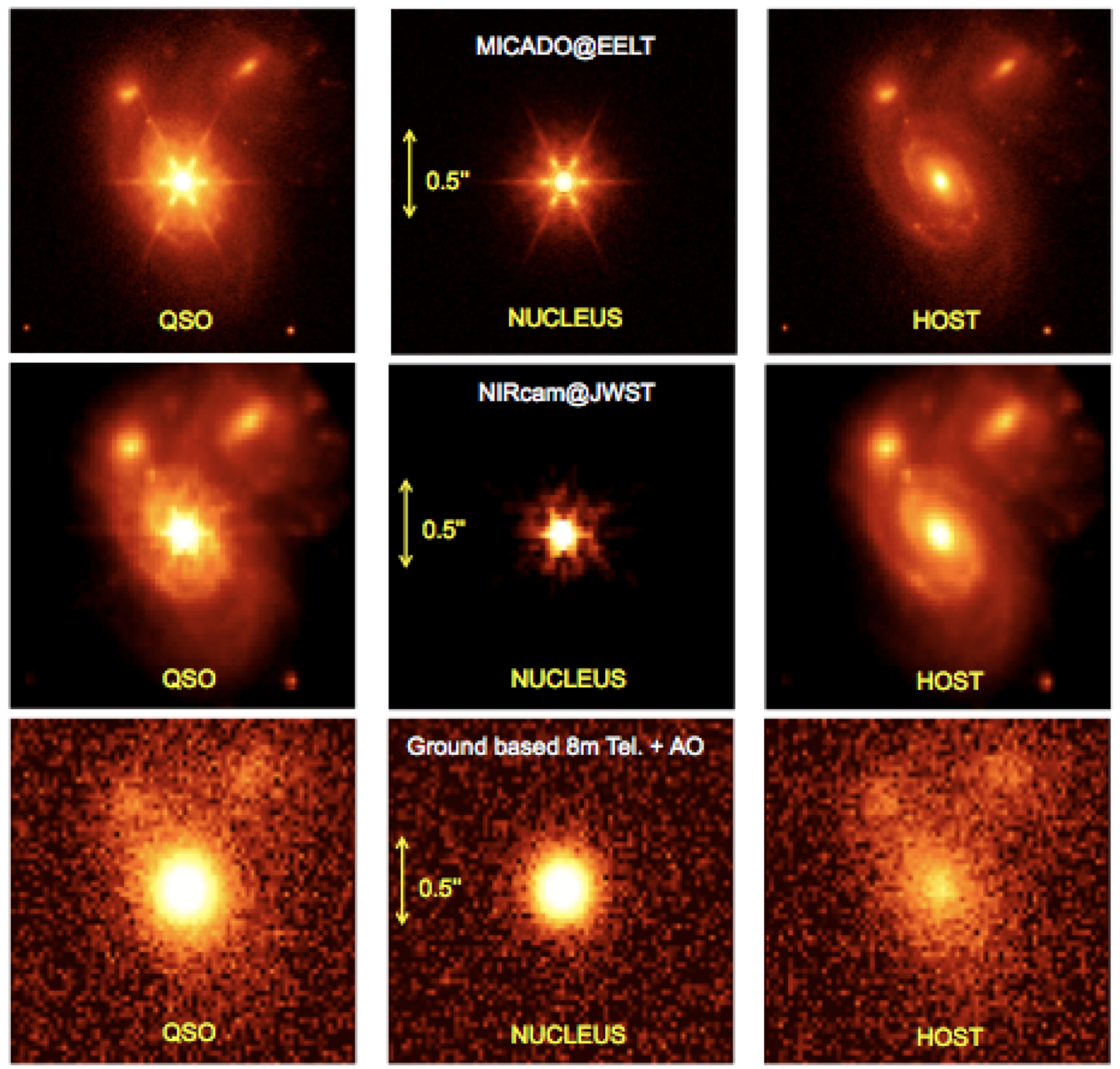

Figure 4. Simulated images (Ks band; $2 \mathrm{~h}$ exp) of a QSO at $\mathrm{z}=1$ as imaged by MICADO@EELT (top) and compared with those expected using NIRcam@JWST (middle) and by a ground-based 8m telescope equipped with AO (alike MAD@VLT; bottom). The QSO (left panels) is composed by a nucleus (middle panels) of Ks=18 and a host galaxy (right panels) of $\mathrm{Ks}=19$ and size Re=0.3". For the host galaxy the image template of NGC 6050 (the brightest galaxy and its companions) is used. 

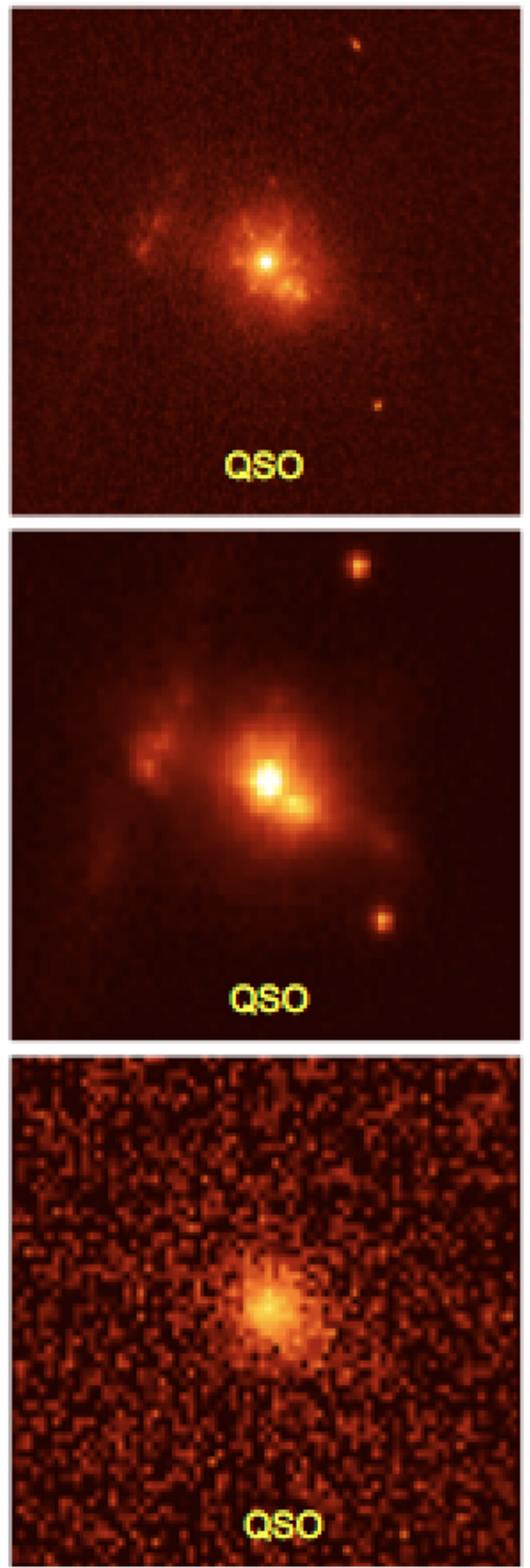
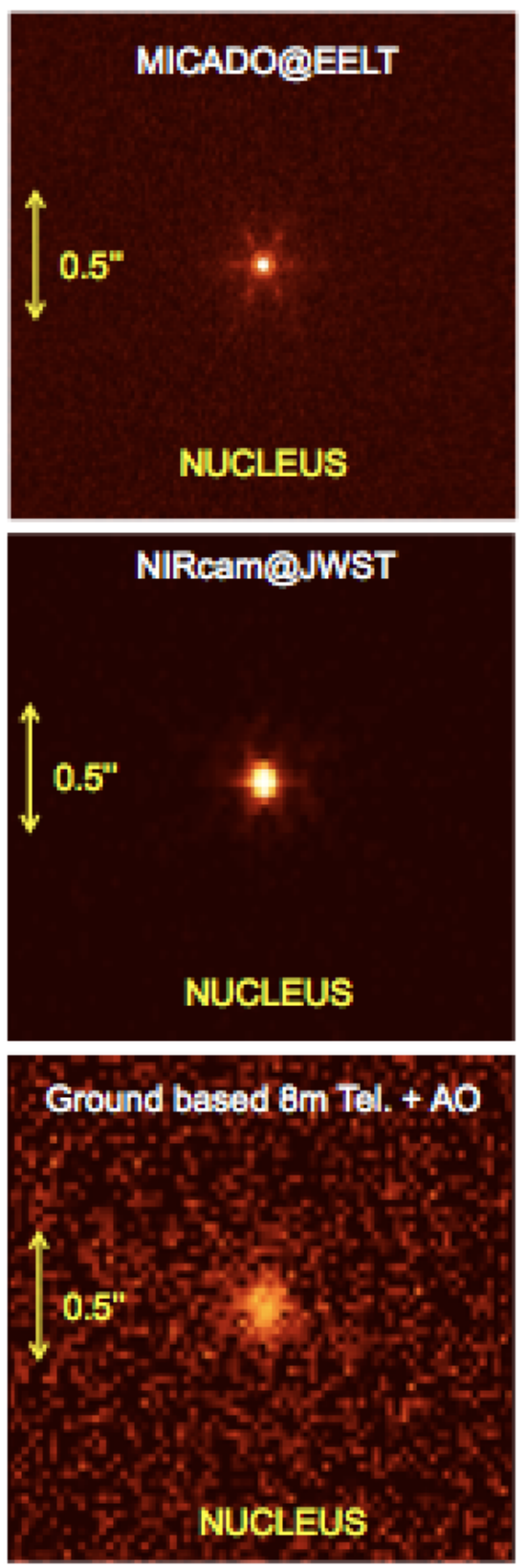
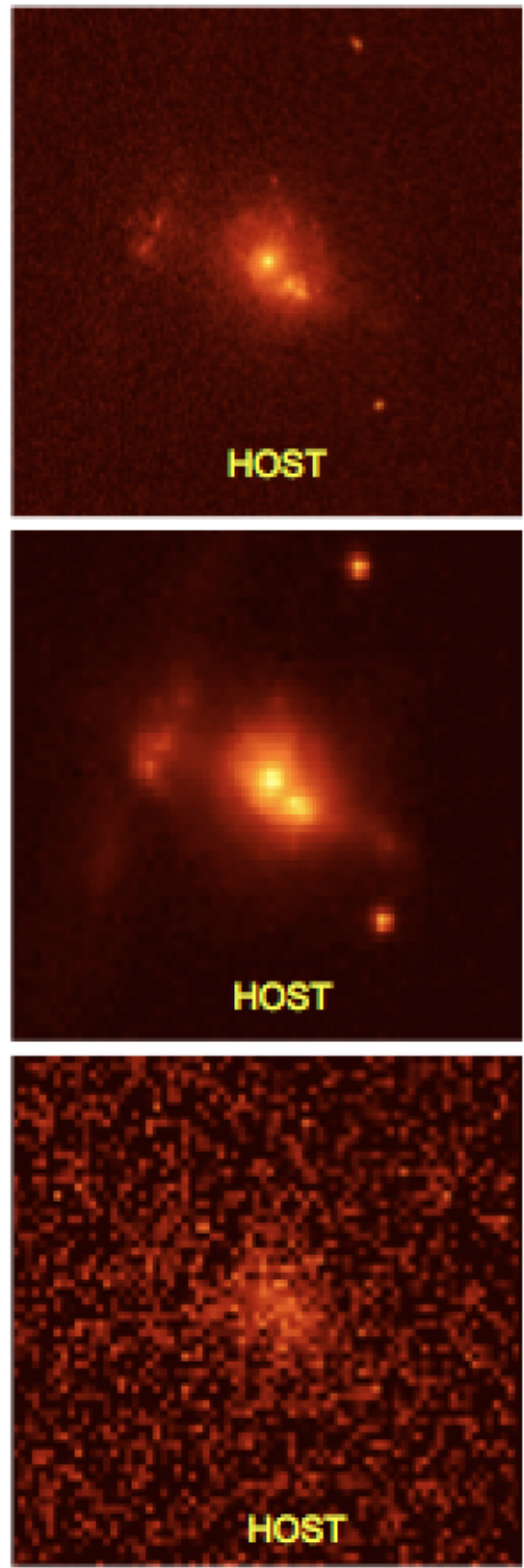

Figure 5. Similar to Fig. 1 but for a QSO at $\mathrm{z}=3$ with 3 hours of exposure time. In this case the nucleus of the QSO is $\mathrm{Ks}=21$, the host galaxy is $\mathrm{Ks}=21$ and Re=0.1". For the host galaxy the image template of NGC 1309 was used with in addition two faint companions $(\mathrm{Ks}=21.5)$ at a distance of $0.15 \%$ and 0.35 " from the nucleus. 